Review Article

\section{Neutrophils, NETs, NETosis and their paradoxical roles in COVID-19}

\author{
Al-Anazi KA ${ }^{1 *}$, Al-Anazi WK ${ }^{2}$ and Al-Jasser $\mathrm{AM}^{3}$ \\ 'Department of Hematology and Hematopoietic Stem Cell Transplantation, Oncology Center, King \\ Fahad Specialist Hospital, Dammam, Saudi Arabia \\ ${ }^{2}$ Section of Cytogenetics, Department of Pathology, King Fahad Specialist Hospital, Dammam, \\ Saudi Arabia \\ ${ }^{3}$ Department of Research and Studies, General Directorate of Health Affairs in Riyadh Region, \\ Ministry of Health, Riyadh 12822, Saudi Arabia
}

\section{Abstract}

The pandemic of COVID-19 has adversely affected the world in many aspects. The health and economic sectors suffer most of the repercussions of this disease. The search for a cure for this rapidly spreading virus which is causing massive life losses worldwide requires clear understanding of the immunopathogenesis of this virus so as to develop pinpointed targeted therapies rather than relying mainly on supportive care measures and drug repurposing to fight this life-threatening virus infection.

Neutrophils, neutrophil extracellular traps (NETs), and NETosis are not well studied not only in COVID-19, but also in coroviruses in general. The review will shed lights on the functions of neutrophils, NETs, and NETosis in various infectious complications as well as in sepsis and acute lung conditions in an attempt to understand their actual roles and in order to help in designing targeted therapies in the near future.

\section{Introduction}

In late December 2019, an unprecedented outbreak of pneumonia emerged in Wuhan City, Hubei Province in China. The infection was caused by a novel beta coronavirus which was initially called severe acute respiratory syndrome (SARS-CoV-2), then it was named coronavirus disease-2019 (COVID-19) by the world health organization (WHO) [1-4]. Due to the continuous and rapid rise in the incidence of COVID-19 worldwide and due to the massive human life losses as well as the huge impact of the infection on world economy, the WHO declared COVID-19 a pandemic on March $11^{\text {th }}, 2010[3-5]$.

The incubation period ranges between 2 and 14 days $[3,4]$. The clinical manifestations of COVID-19 include: fever, cough, shortness of breath, sore throat, fatigue, nausea, vomiting, and diarrhea. However, the illness may be complicated by: severe pneumonia, acute respiratory distress syndrome (ARDS), and respiratory failure; acute cardiac decompensation, arrhythmias, and heart failure; secondary bacterial infection; acute renal and liver dysfunction followed by multiorgan failure; sepsis and septic shock; and death [1,35]. Although the detailed immunopathogenetic mechanisms have not been fully elucidated, the main pathological findings

\section{More Information}

*Address for Correspondence: Dr. Khalid Ahmed Al-Anazi, Consultant, Hemato-Oncologist and Chairman, Department of Hematology and Hematopoietic Stem Cell Transplantation, Oncology Center, King Fahad Specialist Hospital, P.O. Box: 15215, Dammam 31444, Saudi Arabia, Tel: 966-03-8431111;

Fax: 966-13-8427420;

Email: kaa_alanazi@yahoo.com

Submitted: 04 May 2020

Approved: 09 May 2020

Published: 11 May 2020

How to cite this article: Al-Anazi KA, Al-Anazi WK, Al-Jasser AM. Neutrophils, NETs, NETosis and their paradoxical roles in COVID-19. J Stem Cell Ther Transplant. 2020; 4: 003-010.

DOI: 10.29328/journal.jsctt.1001020

Copyright: @ 2020 Al-Anazi KA, et al. This is an open access article distributed under the Creative Commons Attribution License, which permits unrestricted use, distribution, and reproduction in any medium, provided the original work is properly cited.

Keywords: Neutrophils; Neutrophil extracellular traps; NETosis; COVID-19; Aute respiratory distress syndrome; Respiratory failure

Check for updates

OPEN ACCESS that have been described include: diffuse alveolar damage in the lungs manifested by severe pneumonia; immune dysregulation; infection of the cells expressing the surface markers angiotensin converting enzyme (ACE)-2 and TMPRSS2 protein; recruitment of several inflammatory and immune cells including monocytes, macrophages, T-lymphocytes, neutrophils, and B-lymphocytes; and massive production of inflammatory cytokines and chemokines $[2,6]$.

Unfortunately, no specific antiviral treatment is recommended and there is no available vaccine so far $[1,5,7]$. The available therapeutic interventions include: (1) symptomatic measures and supportive care; (2) administration of oxygen via mask, non-invasive ventilation, endotracheal intubation and mechanical ventilation; (3) management of septic shock and secondary bacterial infections; (4) several antiviral and anti-inflammatory drugs have been repurposed and these include: corticosteroids, interferons, chloroquine, ribavirin, lopinavir, ritonavir, remdesivir, and arbidol; (5) monoclonal antibodies such as tocilizumab which is used in the treatment of cytokine release syndrome to inhibit interleukin (IL)-6; (6) ACE inhibitors; (7) Chinese traditional medicines; (8) convalescent serum or plasma containing virus antibodies; (9) auxiliary blood purification therapy; and (10) 
cellular therapies including the use of mesenchymal stem cells (MSCs) [1,2,5-7]. However, studies have shown that combination of several therapeutic modalities appear to be more successful than using single agents $[1,5,7]$.

\section{Neutrophils}

Neutrophils are classically considered as essential players in host defense against invading pathogens [8]. They are: the most abundant leukocytes in the peripheral circulation, the key components of the effector and regulatory mechanisms of both the innate and adaptive immune responses, and the first cells to migrate to the sites of infection and sterile inflammation in order to exhibit a wide range of sophisticated functions including NETosis, which is the release of neutrophil extracellular traps (NETs), and killing microorganisms by phagocytosis [9-14]. The short half-life of neutrophils in the circulation, which is approximately 4 hours, is balanced by their continuous and tightly controlled release from the bone marrow [12]. Neutrophils reaching the circulation are: equipped with the proteins that are required to kill microorganisms and directed by cytokines into the infected cells [15]. In response to infection, polymorphonuclear leukocytes (PMNLs) are recruited to the sites of infection and they employ the following 3 major strategies to fight various microbes: phagocytosis, degranulation, and NETosis [16-18].

Neutrophils have various types of granules that contain hundreds of proteins, enzymes and other substances with important effects on innate and adaptive immune responses and these include: $\alpha$-defensins, lactoferrin, neutrophil elastase, myeloperoxidase, citrullinated histone $\mathrm{H} 3$, human cathelicidin antimicrobial peptide LL-37, and human cathelicidin cationic antimicrobial protein-18 (hCAP18) [9,11]. Neutrophils target pathogens by diverse mechanisms that include: phagocytosis, pinocytosis, cytolysis, cytotoxicity, NETosis with the extrusion on an extracellular chromatin meshwork, generation of reactive oxygen species (ROS), and release of microbicidal molecules from cytoplasmic granules [12,15,19-22]. During infection, neutrophils can undergo beneficial suicide resulting in the production or release of NETs in order to combat invasion by pathogens [13].

Several studies have shown that, during overwhelming infections and severe sepsis, not only that neutrophils become dysfunctional or even paralyzed but also that their antimicrobial arsenal may contribute to further tissue damage and organ failure so that the host becomes unable to contain or eliminate the infection [8,23-26]. In patients with severe inflammation, infectious complications may develop even in the presence of neutrophilia as dysfunction of neutrophils will ultimately result in inability of the host to clear the existing infection [26]. Hence, in immunocompromised patients having neutropenia with severe sepsis and overwhelming infections, host immunity can be boosted further by donor granulocyte transfusions and intravenous immunoglobulins [27-30].
New concepts on neutrophils and their functions: Traditionally, neutrophils have been considered as shortlived, relatively homogeneous population and as terminally differentiated cells that do not recirculate [31]. However, recent studies have shown that neutrophils may differentiate into distinct subsets defined by: specific phenotype and functional profile with well-defined genomic and molecular markers under certain physiological as well as pathological circumstances that include: cancer, sepsis, trauma, ischemic reperfusion injury in addition to ageing and transformation of neutrophils [31-34]. As early as the year 1920, it was realized that circulating neutrophils could show significant differences in parameters such as phagocytosis, protein synthesis, and oxidative metabolism [31]. Additionally, neutrophils can exhibit reverse transmigration and reenter the circulation after shifting their phenotype towards a proinflammatory state with longer life span of about 5.4 days and this may ultimately lead to dissemination of systemic inflammation [31].

Studies have shown that neutrophils are involved in: (1) activation and maturation of macrophages, monocytes and dendritic cells (DCs), (2) regulation of T-cell immune responses against various pathogens and tumor antigens, and (3) complex bidirectional interaction or crosstalk with macrophages, T-lymphocytes, natural killer cells, MSCs, platelets, and B-lymphocytes [33-35]. Many of the effector functions of neutrophils are regulated by a series of immunoreceptors on the plasma membranes [36]. Progress in understanding the heterogeneity and plasticity of neutrophils, determination of specific neutrophil subtypes, and the identification of the interactions mediated by the immunoreceptors of neutrophils may be helpful in the diagnosis of specific diseases and in the development of novel therapeutic interventions $[31,32,36]$.

Neutrophils in viral infections: Neutrophils are capable of recognizing viruses via viral pathogen-associated molecular patterns (PAMPs) and they respond to viruses with specific effector functions [39]. The number of neutrophils in the lower respiratory tract in patients with severe pneumonia correlates with disease activity but may contribute to ALI and other detrimental effects to the host $[37,38]$. In a mouse model, systemic administration of virus analogs or poxvirus infection may induce recruitment of neutrophils to the sites of infection in order to release NETs that can protect host cells from virus infection [39]. Mechanisms by which neutrophils contribute to clearance of viral pathogens include: virus internalization and killing; interaction with other immune cell populations; release of cytokines, chemokines, and antimicrobial components; viral sensing by cytosolic RNA helicases; and NET formation which may further mediate antiviral defense by trapping and inactivating viruses [38]

The cytokines induced by PAMPs and produced by leukocytes are predominantly inflammatory and they include: tumor necrosis factor (TNF)- $\alpha$, IL- 6 , and IL-1 components $[38,40]$. Production or release of inflammatory cytokines and 
chemokines is the siren of neutrophil recruitment to the sites of infection and inflammation. However, in patients having severe pneumonia or sepsis, accumulation of neutrophils in the microcirculation leads to excessive cytokine release or cytokine storm that can lead to deleterious complications and poor clinical outcome [41-43]. Therefore, neutrophils may be a keystone species in determining the outcome of viral disease $[37,38]$.

Recently, it has been shown that viruses act as triggers of the process of NETosis [44-47]. However, virus-induced NETosis can act as a double-edged sword: on one hand making mechanical entrapment of the virus while on the other hand causing harm by the release of NETs triggered by the inflammatory and immunological reactions. Additionally, virus-induced NETs can circulate in an uncontrolled manner leading to an extreme systemic response manifested by production of cytokines, chemokines, and immune complexes that favor inflammation [44]. Neutrophils as well as NET formation play important roles in Dengue virus infections in humans $[48,49]$. In addition to the induction of thrombosis, NETs may acquire proinflammatory roles and cause damage to the activated human endothelial cells [50].

\section{Immunological and hematological changes in COVID-19}

Several studies on COVID-19 have shown the following abnormalities: (1) dysregulation of immune responses; (2) functional exhaustion of cytotoxic lymphocytes; and (3) abnormal peripheral blood picture including: low blood counts of lymphocytes, monocytes, eosinophils, and basophils; low hemoglobin level; low platelet count; leukocytosis or leukopenia; high neutrophil: lymphocyte ratio (NLR); and high monocyte: lymphocyte ration [51-55]. Also studies have shown that high NLR and lymphopenia are independent risk factors for: disease severity, poor clinical outcome, and mortality [51,53,54].

\section{NETs}

NETs are threads or web-like structures of unique extracellular DNA framework, decorated with antimicrobial peptides or proteins released from cell death of activated neutrophils to trap, degrade, fight, and kill pathogens [14,56-59]. So, NETs are extracellular structures composed of chromatin and granule proteins that bind and kill microorganisms $[15,19]$. NETs arise from neutrophils that have activated a cell death program called NETosis or NET cell death $[19,59]$. Upon stimulation, nuclei of neutrophils lose their shape, the nuclear envelope and granule membrane disintegrate and finally NETs are released when the cell membrane breaks [15]. NETs; which trap, immobilize, and then destroy microbes; are one of the most important discoveries in immunological research in recent years $[10,60]$. Historically: NETs were first reported to kill bacteria by degrading their virulence factor by Brinkmann V. et al in 2004. Although NET formation was discovered in 1996, the term NETosis was first coined by Steinberg and Grinstein to describe suicidal NETosis [10,17,18,61-64].

It is unknown whether NET formation takes place in bloodstream or in body tissues [56]. Pathogens are trapped, immobilized within viscous web-like structures and influenced by high concentrations of antimicrobial compounds such as: neutrophil elastase, histones, and myeloperoxidase [57]. NETs; which are composed of degraded chromatin and granule of neutrophil origin; may play important roles in innate immunity against microbial infections [9,58]. Factors that have been demonstrated to influence NET pathways include: (1) internal factors such as production of ROS and activation of transcription factor, and (2) external factors such as alkaline PH and hypertonic conditions [56]. During sepsis, NETs promote pyroptosis or regulated cell death of macrophages [16]. In patients with septic shock, unstimulated NET formation and nuclease activity are reduced [20].

The formation of NETs can be influenced by: (1) microorganisms such as bacteria, viruses, fungi, and parasites; (2) cytokines such as IL-8 and TNF- $\alpha$; (3) antimicrobials such as amoxicillin; and (4) chemicals such as calcium ionophore A23187 and phorbol myristate acetate (PMA) [10,13,57]. The key enzymes that are involved in NET formation include: (1) neutrophil elastase which degrades intracellular proteins and triggers nuclear disintegration; (2) peptidyl arginine deiminase type 4 (PAD-4) which citrullinates histones to facilitate the decompensation and release of chromosomal DNA; (3) gasdermin D which generates pores in the membranes of neutrophils thereby facilitating cell membrane rupture and expulsion of DNA and associated molecules; and (4) myeloperoxidase granule enzyme $[21,65,66]$.

Antimicrobial activity of NETs can be measured by different methods including: (1) induction of the formation of NETs then addition of microbe and finally assessment of the number of surviving bacteria after an incubation period, and (2) measuring microbial killing by blocking NET components with antibodies or cation chelators such as zinc [18]. Statins have been found to enhance the formation of phagocyte extracellular traps [67].

NETs can inactivate virulence factors or microbial proteins that modify the function of host cells $[18,61]$. Fully hydrated NETs have a cloud-like appearance and they occupy a space which is 10 to 15 times larger than the volume of the cells they originate from [18]. Identification or detection of NETs might serve as a biomarker that could help in identifying individuals at high risk of developing consequences of acute lung injury (ALI) and acute kidney injury (AKI) [68,69]. Methods that can be used to quantify or visualize NETs include: high resolution scanning by electron microscopy; intravital photon microscopy; flowcytometry; fluorescent labelling of microorganisms by direct visualization; immunostaining and automated microscopy using computer-assisted analysis 
to quantify NETs from fluorescence images; and machine learning using conventional neural networks $[10,17,18,61$ 64].

The dark side of NETs: In addition to their antimicrobial actions, NETs have a dark side reflected by their involvement in certain diseases or complications such as: (1) autoimmunity and autoimmune disorders such as systemic lupus erythromatosis, rheumatoid arthritis, and psoriasis; (2) pregnancy associated disorders such as preeclampsia; (3) cystic fibrosis; (4) coagulopathy and thrombosis; (5) periodontitis; and (6) tissue injuries [10,13,14,18,50,65,7579]. NETs have prothrombotic properties by stimulation of fibrin deposition and increased NET levels correlate with larger infarct size and predict major cardiovascular complications [78]. Additionally, excessive NET formation can trigger a cascade of inflammatory reactions that destroy surrounding tissue, facilitate microthrombi and result in permanent damage to the pulmonary, cardiovascular and renal systems [21]. NETs can exert direct cytotoxic effects on lung epithelium and endothelium and excessive production of NETs has been found in patients with ALI and pneumonia. Thus, NET formation can exert positive as well as negative influences on multiple lung pathologies $[80,81]$.

In patients with diabetes mellitus, hyperglycemia induces or boosts NET formation and this may cause direct damage to endothelial cells and may predispose to complications such as diabetic retinopathy and diabetic wounds $[10,78]$. In patients with severe influenza A virus infection, high levels of NETs contribute to lung injury, correlate with disease severity and imply poor prognosis [82]. In a model of influenza A virus $\left(\mathrm{H}_{1} \mathrm{~N}_{1}\right)$ infection, excessive neutrophils and NETs might lead to: ALI, ARDS, and pneumonitis with alveolar-capillary damage [83]. Nicotine has been found to induce NETs which may contribute to smoking-related lung diseases [84].

\section{NETosis}

NETosis, a recently described neutrophil function, leads to the release of NETs in response to various stimuli and it represents the most dramatic stage in the process of cell death [11,14]. During NETosis, PMNLs undergo specific morphological changes that include: chromatin condensation leading to loss of the lobulated nucleus, disintegration of intracellular membranes that allows chromatin and extracellular protein to mix, and the release of chromatin filaments decorated with PMNL proteins derived from several cell compartments into the extracellular medium [14].

In NETosis, the following enzymes, chemicals, and signaling pathways are involved: (1) neutrophil elastase, (2) myeloperoxidase, (3) PAD-4, (4) PMA, (5) nicotinamide adenine dinucleotide phosphate (NADPH) which generates ROS, (6) mitogen activated protein kinase/extracellular signal-regulated kinase (MAPK/ERK) pathway, (7) Tolllike receptors (TLRs), and (8) autophagy pathway. Also, the following steps or changes take place during NETOSIS: activated neutrophils flatten and lose lobes of their nuclei, chromatin becomes condensed, nuclear detachment of the inner and outer membranes, separation of granules, the nuclear envelope breaks into pieces, and the cells roundup until the cell membrane ruptures and ejects the inner contents into the extracellular space forming NETs $[15,17,18,74]$.

There are 2 types of NETosis [17,74]. The first type is suicidal NETosis which is slow, takes hours, and is induced by chemical stimuli such as PMA. In this type of NETosis, the following take place: occurrence of morphological changes in activated neutrophils, the release of NETs results in neutrophil death through a different pathway than apoptosis or necrosis, and the intracellular NET formation is followed by rupture of plasma membrane releasing the contents into the extracellular space thus forming NETs. The second type is vital NETosis which is rapid, takes minutes, and is induced by bacteria and other pathogens. In this type of NETosis: stimulated neutrophils remain active and functional following NET formation, the process results in blebbing of the nucleus to produce a DNA-filled vesicle that is exocytosed thus leaving the plasma membrane intact, and neutrophils can continue to phagocytose and kill microbes after NETosis [17,74].

\section{Neutrophils, NETs and NETosis in COVID-19}

\section{The pathological consequences of SARS-CoV} infection in the lung include: (1) the virus applies several mechanisms to overcome the immune response including: inhibition of the rapid expression on type 1 interferon (IFN1), intervention with IFN-signaling through inhibition of STAT 1 phosphorylation, and immune exhaustion through exaggerated and prolonged IFN-1 production by plasmacytoid DCs; and (2) influx of activated neutrophils and inflammatory monocytes/macrophages resulting in ARDS and cytokine storm thus weakening the immune system through IFN-1 mediated T-cell apoptosis. SARS-CoV2 causing COVID-19 is expected to have the same or at least similar consequences on the immune system as SARS-CoV due to similarities between the 2 coronaviruses [85]. So, it is possible that: (1) excessive recruitment of various immune cells such as neutrophils, macrophages, monocytes, DCs, and T-lymphocytes; (2) NETs; and (3) NETosis may be responsible for many of the serious complications of COVID-19 such as: ARDS, cytokine storm, thromboembolic complications, acute organ dysfunction, and multiorgan failure $[44,85,86]$. In patients with COVID-19, high levels of NETs have been documented and it has been found that NETs may contribute to: cytokine release, ARDS, respiratory failure, as well as disseminated inflammation and microvascular thrombosis [87].

\section{Possible therapies and therapeutic targets for COVID-19}

Targeting upregulation or downregulation of NETs with destruction or protection of already formed NETs may become a valuable therapeutic intervention in patients 
having severe pneumonia or ARDS $[80,88]$. As circulating NETs may be directly responsible for orchestrating ALI and ARDS, inhibition of NETosis may become valuable in reducing inflammation and organ damage [68]. In patients with respiratory syncytial virus infection, neutrophils could limit viral replication and spread by stimulating antiviral adaptive and immune responses [89]. Immune mediators such as: (1) GTS-21; the selective $\alpha 7$ Ach receptor agonist that has the same inflammatory modulation effects as nicotine but without the risk of addiction and other side effects; and (2) platelet-derived acting factor-acetylhydroxylase (RAF-AH) may become widely available for the treatment of cytokine storm associated with viral pneumonia [40].

Prostaglandin-E2 has been found to inhibit NET formation [90]. Probiotic Lactobacillus rhamnosus strain BB has potent antioxidant activity and can reduce the phagocytic function of neutrophil and inhibit NET formation [62]. The combination of zinc and pyrithione has been found to inhibit the replication of SARS-CoV (SARS coronavirus) [91]. In patients with COVID-19, the ability to form NETs may contribute to organ damage and increased mortality. Hence, targeting NETs directly or indirectly with the existing drugs may reduce the clinical severity of COVID-19 infection [21]. Also, treatments that inhibit viral replication or target regulation of the dysfunctional immune reactions may offer synergistic effects in order to block viral pathologies at multiple levels [6].

\section{Conclusion and future directions}

Many of the complications of COVID-19 such as: respiratory distress and failure; multiorgan dysfunction including cardiac decompensation; thromboembolic phenomena, the associated cytokine storm as well as the poor outcome encountered in cigarette smokers and in patients with diabetes mellitus can well be explained by the dysfunctional neutrophils and their products. Apparently the functions of neutrophils, NETs, and NETosis are not well characterized in COVID-19 due to the relative lack of studies on this aspect of the disease. As depicted from other viral infections that involve the lungs and cause serious complications, the roles of neutrophils, NETs, and NETosis seem to be paradoxical under certain circumstances. Therefore, further studies are needed in this field. These studies should focus not only on the numbers of neutrophils but also on their functions, subsets, life span of each subset, as well as migration to tissues and organs affected by infection, inflammation and other injuries. Such studies are likely to help in developing more efficacious therapeutic interventions that can bring cure to this devastating, widely spreading and life-threatening viral illness.

\section{References}

1. Wang L, Wang $Y$, Ye D, Liu Q. Review of the 2019 novel coronavirus (SARS-CoV-2) based on current evidence. Int J Antimicrob Agents. 2020; 105948.

Pubmed: https://www.ncbi.nlm.nih.gov/pubmed/32201353
2. Contini C, Di Nuzzo M, Barp N, Bonazza A, De Giorgio R, et al. The novel zoonotic COVID-19 pandemic: An expected global health concern. J Infect Dev Ctries. 2020; 14: 254-264.

Pubmed: https://www.ncbi.nlm.nih.gov/pubmed/32235085

3. Park SE. Epidemiology, virology, and clinical features of severe acute respiratory syndrome-coronavirus-2 (SARS-CoV-2; Coronavirus Disease-19). Clin Exp Pediatr. 2020; 63: 119-124.

Pubmed: https://www.ncbi.nlm.nih.gov/pubmed/32252141

4. Sohrabi C, Alsafi Z, O'Neill N, Khan M, Kerwan A, et al. World Health Organization declares global emergency: A review of the 2019 novel coronavirus (COVID-19). Int J Surg. 2020; 76: 71-76.

Pubmed: https://www.ncbi.nlm.nih.gov/pubmed/32112977

5. Tu H, Tu S, Gao S, Shao A, Sheng J. The epidemiological and clinical features of COVID-19 and lessons from this global infectious public health event. J Infect. 2020; S0163-4453(20)30222-X.

Pubmed: https://www.ncbi.nlm.nih.gov/pubmed/32315723

6. Tay MZ, Poh CM, Rénia L, Mac Ary PA, Ng LFP. The trinity of COVID-19: immunity, inflammation and intervention. Nat Rev Immunol. 2020. Pubmed: https://www.ncbi.nlm.nih.gov/pubmed/32346093

7. Yan Y, Shin WI, Pang YX, Meng Y, Lai J, et al. The first 75 days of novel coronavirus (SARS-CoV-2) outbreak: Recent advances, prevention, and treatment. Int J Environ Res Public Health. 2020; 17: E2323. Pubmed: https://www.ncbi.nlm.nih.gov/pubmed/32235575

8. Sônego F, Castanheira FV, Ferreira RG, Kanashiro A, Leite CA, et al Paradoxical roles of the neutrophil in sepsis: protective and deleterious. Front Immunol. 2016; 7: 155.

Pubmed: https://www.ncbi.nlm.nih.gov/pubmed/27199981

9. Shiogama K, Onouchi T, Mizutani Y, Sakurai K, Inada K, et al. Visualization of neutrophil extracellular traps and fibrin meshwork in human fibrinopurulent inflammatory lesions: I. light microscopic study. Acta Histochem Cytochem. 2016; 49: 109-116.

Pubmed: https://www.ncbi.nlm.nih.gov/pubmed/27682014

10. Hasler P, Giaglis $\mathrm{S}$, Hahn $\mathrm{S}$. Neutrophil extracellular traps in health and disease. Swiss Med Wkly. 2016; 146: w14352.

Pubmed: https://www.ncbi.nlm.nih.gov/pubmed/27723901

11. Kaplan MJ, Radic M. Neutrophil extracellular traps: Double-edged swords of innate immunity. J Immunol. 2012; 189: 2689-2695. Pubmed: https://www.ncbi.nlm.nih.gov/pubmed/22956760

12. Knight JS, Carmona-Rivera C, Kaplan MJ. Proteins derived from neutrophil extracellular traps may serve as self-antigens and mediate organ damage in autoimmune diseases. Front Immunol. 2012; 3: 380. Pubmed: https://www.ncbi.nlm.nih.gov/pubmed/23248629

13. Bornhöfft KF, Viergutz T, Kühnle A, Galuska SP. Nanoparticles equipped with $\alpha 2,8$-linked sialic acid chains inhibit the release of neutrophil extracellular traps. Nanomaterials. 2019; 9: E610. Pubmed: https://www.ncbi.nlm.nih.gov/pubmed/31013834

14. Barrientos L, Marin-Esteban V, de Chaisemartin L, Le-Moal VL, Sandré $\mathrm{C}$, et al. An improved strategy to recover large fragments of functional human neutrophil extracellular traps. Front Immunol. 2013; 4: 166. Pubmed: https://www.ncbi.nlm.nih.gov/pubmed/23805143

15. Fuchs TA, Abed U, Goosmann C, Hurwitz R, Schulze I, et al. Novel cell death program leads to neutrophil extracellular traps. J Cell Biol. 2007; 176: 231-241.

Pubmed: https://www.ncbi.nlm.nih.gov/pubmed/17210947

16. Chen L, Zhao Y, Lai D, Zhang P, Yang Y, et al. Neutrophil extracellular traps promote macrophage pyroptosis in sepsis. Cell Death Dis. 2018; 9: 597.

Pubmed: https://www.ncbi.nlm.nih.gov/pubmed/29789550 
17. Alasmari SZ. In vivo imaging of neutrophil extracellular traps (NETs) Visualization methods and outcomes. Biomed Res Int. 2020; 2020: 4192745.

Pubmed: https://www.ncbi.nlm.nih.gov/pubmed/32090090

18. Brinkmann V, Zychlinsky A. Neutrophil extracellular traps: Is immunity the second function of chromatin? J Cell Biol. 2012; 198: 773-783. Pubmed: https://www.ncbi.nlm.nih.gov/pubmed/22945932

19. Palmer LJ, Cooper PR, Ling MR, Wright HJ, Huissoon A, et al. Hypochlorous acid regulates neutrophil extracellular trap release in humans. Clin Exp Immunol. 2012; 167: 261-268.

Pubmed: https://www.ncbi.nlm.nih.gov/pubmed/22236002

20. Cox LE, Walstein K, Völlger L, Reuner F, Bick A, et al. Neutrophi extracellular trap formation and nuclease activity in septic patients. BMC Anesthesiol. 2020; 20: 15.

Pubmed: https://www.ncbi.nlm.nih.gov/pubmed/31931719

21. Barnes BJ, Adrover JM, Baxter-Stoltzfus A, Borczuk A, CoolsLartigue J, et al. Targeting potential drivers of COVID-19: Neutrophil extracellular traps. J Exp Med. 2020; 217: e20200652.

Pubmed: https://www.ncbi.nlm.nih.gov/pubmed/32302401

22. Niedźwiedzka-Rystwej P, Repka W, Tokarz-Deptuła B, Deptuła W. In sickness and in health - how neutrophil extracellular trap (NET) works in infections, selected diseases and pregnancy. J Inflamm. 2019; 16: 15. Pubmed: https://www.ncbi.nlm.nih.gov/pubmed/31297037

23. Zhang F, Liu AL, Gao S, Ma S, Guo SB. Neutrophil dysfunction in sepsis. Chin Med J. 2016; 129: 2741-2744.

Pubmed: https://www.ncbi.nlm.nih.gov/pubmed/27824008

24. Shen XF, Cao K, Jiang JP, Guan WX, Du JF. Neutrophil dysregulation during sepsis: an overview and update. J Cell Mol Med. 2017; 21: 1687-1697.

Pubmed: https://www.ncbi.nlm.nih.gov/pubmed/28244690

25. Mortaz E, Alipoor SD, Adcock IM, Mumby S, Koenderman L. Update on neutrophil function in severe inflammation. Front Immunol. 2018; 9: 2171.

Pubmed: https://www.ncbi.nlm.nih.gov/pubmed/30356867

26. Leliefeld PH, Wessels CM, Leenen LP, Koenderman L, Pillay J. The role of neutrophils in immune dysfunction during severe inflammation. Crit Care. 2016; 20: 73.

Pubmed: https://www.ncbi.nlm.nih.gov/pubmed/27005275

27. Al-Jasser AM, Al-Anazi KA. Donor granulocyte transfusions in patients with hematologic malignancies and in recipients of hematopoietic stem cell transplantation. J Stem Cell Biol Transplant. 2019; 3: 1.

28. Drewniak A, Kuijpers TW. Granulocyte transfusion therapy: randomization after all? Haematologica. 2009; 94: 1644-1648. Pubmed: https://www.ncbi.nlm.nih.gov/pubmed/19996116

29. Cui J, Wei X, Lv H, Li Y, Li P, et al. The clinical efficacy of intravenous IgM-enriched immunoglobulin (pentaglobin) in sepsis or septic shock: a meta-analysis with trial sequential analysis. Ann Intensive Care. 2019; 9: 27.

Pubmed: https://www.ncbi.nlm.nih.gov/pubmed/30725235

30. Alejandria MM, Lansang MA, Dans LF, Mantaring JB 3rd. Intravenous immunoglobulin for treating sepsis, severe sepsis and septic shock. Cochrane Database Syst Rev. 2013; CD001090.

Pubmed: https://www.ncbi.nlm.nih.gov/pubmed/11869591

31. Beyrau M, Bodkin JV, Nourshargh S. Neutrophil heterogeneity in health and disease: a revitalized avenue in inflammation and immunity. Open Biol. 2012; 2: 120134.

Pubmed: https://www.ncbi.nlm.nih.gov/pubmed/23226600

32. Silvestre-Roig C, Fridlender ZG, Glogauer M, Scapini P. Neutrophil diversity in health and disease. Trends Immunol. 2019; 40: 565-583. Pubmed: https://www.ncbi.nlm.nih.gov/pubmed/31160207
33. Prame Kumar K, Nicholls AJ, Wong CHY. Partners in crime: neutrophils and monocytes/macrophages in inflammation and disease. Cell Tissue Res. 2018; 371: 551-565.

Pubmed: https://www.ncbi.nlm.nih.gov/pubmed/29387942

34. Scapini $P$, Cassatella MA. Social networking of human neutrophils within the immune system. Blood. 2014; 124: 710-719.

Pubmed: https://www.ncbi.nlm.nih.gov/pubmed/24923297

35. Kumar V, Sharma A. Neutrophils: Cinderella of innate immune system. Int Immunopharmacol. 2010; 10: 1325-1334.

Pubmed: https://www.ncbi.nlm.nih.gov/pubmed/20828640

36. van Rees DJ, Szilagyi K, Kuijpers TW, Matlung HL, van den Berg TK. Immunoreceptors on neutrophils. Semin Immunol. 2016; 28: 94-108. Pubmed: https://www.ncbi.nlm.nih.gov/pubmed/26976825

37. Camp JV, Jonsson CB. A role for neutrophils in viral respiratory disease. Front Immunol. 2017; 8: 550.

Pubmed: https://www.ncbi.nlm.nih.gov/pubmed/28553293

38. Galani IE, Andreakos E. Neutrophils in viral infections: Current concepts and caveats. J Leukoc Biol. 2015; 98: 557-564. Pubmed: https://www.ncbi.nlm.nih.gov/pubmed/26160849

39. Jenne CN, Wong CH, Zemp FJ, McDonald B, Rahman MM, et al. Neutrophils recruited to sites of infection protect from virus challenge by releasing neutrophil extracellular traps. Cell Host Microbe. 2013; 13: 169-180.

Pubmed: https://www.ncbi.nlm.nih.gov/pubmed/23414757

40. D'Elia RV, Harrison K, Oyston PC, Lukaszewski RA, Clark GC Targeting the cytokine storm for therapeutic benefit. Clin Vaccine Immunol. 2013; 20: 319-327.

Pubmed: https://www.ncbi.nlm.nih.gov/pubmed/23283640

41. Bordon J, Aliberti S, Fernandez-Botran R, Uriarte SM, Rane MJ, et al. Understanding the roles of cytokines and neutrophil activity and neutrophil apoptosis in the protective versus deleterious inflammatory response in pneumonia. Int J Infect Dis. 2013; 17: e76-83. Pubmed: https://www.ncbi.nlm.nih.gov/pubmed/23069683

42. McDonald B, Kubes P. Chemokines: sirens of neutrophil recruitmentbut is it just one song? Immunity. 2010; 33: 148-149.

Pubmed: https://www.ncbi.nlm.nih.gov/pubmed/20732637

43. McDonald B, Urrutia R, Yipp BG, Jenne CN, Kubes P. Intravascular neutrophil extracellular traps capture bacteria from the bloodstream during sepsis. Cell Host Microbe. 2012; 12: 324-333.

Pubmed: https://www.ncbi.nlm.nih.gov/pubmed/22980329

44. Mozzini C, Girelli D. The role of neutrophil extracellular traps in COVID-19: Only an hypothesis or a potential new field. Thrombosis Res. 2020; 26-27.

Pubmed: https://www.ncbi.nlm.nih.gov/pubmed/32360977

45. Hiroki $\mathrm{CH}$, Toller-Kawahisa JE, Fumagalli MJ, Colon DF, Figueiredo LTM, et al. Neutrophil extracellular traps effectively control acute Chikungunya virus infection. Front Immunol. 2020; 10: 3108. Pubmed: https://www.ncbi.nlm.nih.gov/pubmed/32082301

46. Muraro SP, De Souza GF, Gallo SW, Da Silva BK, De Oliveira SD, et al. Respiratory syncytial virus induces the classical ROS-dependent NETosis through PAD-4 and necroptosis pathways activation. Sci Rep. 2018; 8: 14166.

Pubmed: https://www.ncbi.nlm.nih.gov/pubmed/30242250

47. Schönrich G, Raftery MJ. Neutrophil extracellular traps go viral. Front Immunol. 2016; 7: 366 .

Pubmed: https://www.ncbi.nlm.nih.gov/pubmed/27698656

48. Opasawatchai A, Amornsupawat $\mathrm{P}$, Jiravejchakul N, Chan-In W, Spoerk NJ, et al. Neutrophil activation and early features of NET 
formation are associated with Dengue virus infection in human. Front Immunol. 2019; 9: 3007.

Pubmed: https://www.ncbi.nlm.nih.gov/pubmed/30687301

49. Schulz C, Gabriel G, von Köckritz-Blickwede M. Detrimental role of neutrophil extracellular traps during Dengue virus infection. Trends Immunol. 2020; 41: 3-6.

Pubmed: https://www.ncbi.nlm.nih.gov/pubmed/31791719

50. Borregaard N. Neutrophils, from marrow to microbes. Immunity. 2010; 33: 657-670.

Pubmed: https://www.ncbi.nlm.nih.gov/pubmed/21094463

51. Qin C, Zhou L, Hu Z, Zhang S, Yang S, et al. Dysregulation of immune response in patients with COVID-19 in Wuhan, China. Clin Infect Dis. 2020. ciaa248.

Pubmed: https://www.ncbi.nlm.nih.gov/pubmed/32161940

52. Sun $\mathrm{S}$, Cai $X$, Wang H, He G, Lin $Y$, et al. Abnormalities of peripheral blood system in patients with COVID-19 in Wenzhou, China. Clin Chim Acta. 2020. 507: 174-180.

Pubmed: https://www.ncbi.nlm.nih.gov/pubmed/32339487

53. Yang AP, Liu JP, Tao WQ, Li HM. The diagnostic and predictive role of NLR, d-NLR and PLR in COVID-19 patients. Int Immunopharmacol. 2020; 84: 106504.

Pubmed: https://www.ncbi.nlm.nih.gov/pubmed/32304994

54. Liu Y, Du X, Chen J, Jin Y, Peng L, et al. Neutrophil-to-lymphocyte ratio as an independent risk factor for mortality in hospitalized patients with COVID-19. J Infect. 2020; S0163-4453(20)30208-5.

Pubmed: https://www.ncbi.nlm.nih.gov/pubmed/32283162

55. Zheng M, Gao Y, Wang G, Song G, Liu S, et al. Functional exhaustion of antiviral lymphocytes in COVID-19 patients. Cell Mol Immunol. 2020; 17: 533-535.

Pubmed: https://www.ncbi.nlm.nih.gov/pubmed/32203188

56. Ravindran M, Khan MA, Palaniyar N. Neutrophil extracellular trap formation: Physiology, pathology, and pharmacology. Biomolecules. 2019; 9: E365.

Pubmed: https://www.ncbi.nlm.nih.gov/pubmed/31416173

57. Bystrzycka W, Moskalik A, Sieczkowska S, Manda-Handzlik A Demkow $U$, et al. The effect of clindamycin and amoxicillin on neutrophil extracellular trap (NET) release. Cent Eur J Immunol. 2016; 41: 1-5. Pubmed: https://www.ncbi.nlm.nih.gov/pubmed/27095915

58. Onouchi T, Shiogama K, Mizutani Y, Takaki T, Tsutsumi Y. Visualization of neutrophil extracellular traps and fibrin meshwork in human fibrinopurulent inflammatory lesions: III. Correlative light and electron microscopic study. Acta Histochem Cytochem. 2016; 49: 141-147. Pubmed: https://www.ncbi.nlm.nih.gov/pubmed/27917008

59. Remijsen Q, Vanden Berghe T, Wirawan E, Asselbergh B, Parthoens $\mathrm{E}$, et al. Neutrophil extracellular trap cell death requires both autophagy and superoxide generation. Cell Res. 2011; 21: 290-304.

Pubmed: https://www.ncbi.nlm.nih.gov/pubmed/21060338

60. Neubert E, Meyer D, Kruss S, Erpenbeck L. The power from within - Understanding the driving forces of neutrophil extracellular trap formation. J Cell Sci. 2020; 133: jcs241075.

Pubmed: https://www.ncbi.nlm.nih.gov/pubmed/32156720

61. Brinkmann V, Reichard U, Goosmann C, Fauler B, Uhlemann Y, et al. Neutrophil extracellular traps kill bacteria. Science. 2004; 303: 15321535.

Pubmed: https://www.ncbi.nlm.nih.gov/pubmed/15001782

62. Vong L, Lorentz RJ, Assa A, Glogauer M, Sherman PM. Probiotic Lactobacillus rhamnosus inhibits the formation of neutrophil extracellular traps. J Immunol. 2014; 192: 1870-1877.

Pubmed: https://www.ncbi.nlm.nih.gov/pubmed/24465012
63. Naffah de Souza C, Breda LCD, Khan MA, de Almeida SR, Câmara $\mathrm{NOS}$, et al. Alkaline pH promotes NADPH oxidase-independent neutrophil extracellular trap formation: A matter of mitochondrial reactive oxygen species generation and citrullination and cleavage of histone. Front Immunol. 2018; 8: 1849.

Pubmed: https://www.ncbi.nlm.nih.gov/pubmed/29375550

64. Steinberg BE, Grinstein S. Unconventional roles of the NADPH oxidase: Signaling, ion homeostasis and cell death. Sci STKE. 2007; 2007: pe11. Pubmed: https://www.ncbi.nlm.nih.gov/pubmed/17392241

65. Hemmers S, Teijaro JR, Arandjelovic S, Mowen KA. PAD4-mediated neutrophil extracellular trap formation is not required for immunity against influenza infection. PLoS One. 2011; 6: e22043.

Pubmed: https://www.ncbi.nlm.nih.gov/pubmed/21779371

66. Metzler KD, Fuchs TA, Nauseef WM, Reumaux D, Roesler J, et al Myeloperoxidase is required for neutrophil extracellular trap formation: Implications for innate immunity. Blood. 2011; 117: 953-959. Pubmed: https://www.ncbi.nlm.nih.gov/pubmed/20974672

67. Chow OA, von Köckritz-Blickwede M, Bright AT, Hensler ME, Zinkernagel AS, et al. Statins enhance formation of phagocyte extracellular traps. Cell Host Microbe. 2010; 8: 445-454. Pubmed: https://www.ncbi.nlm.nih.gov/pubmed/21075355

68. Pallet N. Neutrophil extracellular traps orchestrate necroinflammation. J Am Soc Nephrol. 2017; 28: 1670-1672.

Pubmed: https://www.ncbi.nlm.nih.gov/pubmed/28232618

69. Masuda S, Nakazawa D, Shida H, Miyoshi A, Kusunoki $Y$, et al. NETosis markers: Quest for specific, objective, and quantitative markers. Clin Chim Acta. 2016; 459: 89-93.

Pubmed: https://www.ncbi.nlm.nih.gov/pubmed/27259468

70. Schneck E, Mallek F, Schiederich J, Kramer E, Markmann M, et al Flow cytometry-based quantification of neutrophil extracellular traps shows an association with hypercoagulation in septic shock and hypocoagulation in postsurgical systemic inflammation-A proof-ofconcept study. J Clin Med. 2020; 9: E174.

Pubmed: https://www.ncbi.nlm.nih.gov/pubmed/31936385

71. de Buhr N, von Köckritz-Blickwede M. How neutrophil extracellular traps become visible. J Immunol Res. 2016; 2016: 4604713. Pubmed: https://www.ncbi.nlm.nih.gov/pubmed/27294157

72. Mohanty T, Sørensen OE, Nordenfelt P. NETQUANT: Automated quantification of neutrophil extracellular traps. Front Immunol. 2018; 8: 1999.

Pubmed: https://www.ncbi.nlm.nih.gov/pubmed/29379509

73. Elsherif L, Sciaky N, Metts CA, Modasshir M, Rekleitis I, et al. Machine learning to quantitate neutrophil NETosis. Sci Rep. 2019; 9: 16891. Pubmed: https://www.ncbi.nlm.nih.gov/pubmed/31729453

74. Held P, Rumble J. Automated analysis of neutrophil NETosis activity using the Lionheart ${ }^{\mathrm{TM}} \mathrm{FX}$ to image and analyze stimulated dHL60 cells. BioTek, Application Notes, Cellular Imaging, Live Cell Image. 2019.

75. Boeltz S, Amini P, Anders HJ, Andrade F, Bilyy R, et al. To NET or not to NET: current opinions and state of the science regarding the formation of neutrophil extracellular traps. Cell Death Differ. 2019; 26: 395-408.

Pubmed: https://www.ncbi.nlm.nih.gov/pubmed/30622307

76. Konig MF, Andrade F. A critical reappraisal of neutrophil extracellular traps and NETosis mimics based on differential requirements for protein citrullination. Front Immunol. 2016; 7: 461.

Pubmed: https://www.ncbi.nlm.nih.gov/pubmed/27867381

77. Sørensen OE, Borregaard N. Neutrophil extracellular traps - the dark side of neutrophils. J Clin Invest. 2016; 126: 1612-1620.

Pubmed: https://www.ncbi.nlm.nih.gov/pubmed/27135878 
78. Bonaventura A, Vecchié A, Abbate A, Montecucco F. Neutrophil extracellular traps and cardiovascular diseases: An update. Cells. 2020; 9: E231.

Pubmed: https://www.ncbi.nlm.nih.gov/pubmed/31963447

79. Kenny EF, Herzig A, Krüger R, Muth A, Mondal S, et al. Diverse stimul engage different neutrophil extracellular trap pathways. Elife. 2017; 6: e24437.

Pubmed: https://www.ncbi.nlm.nih.gov/pubmed/28574339

80. Twaddell SH, Baines KJ, Grainge C, Gibson PG. The emerging role of neutrophil extracellular traps in respiratory disease. Chest. 2019; 156: 774-782.

Pubmed: https://www.ncbi.nlm.nih.gov/pubmed/31265835

81. Gan T, Yang Y, Hu F, Chen X, Zhou J, et al. TLR3 regulated poly I: C-induced neutrophil extracellular traps and acute lung injury partly through p38 MAP kinase. Front Microbiol. 2018; 9: 3174.

Pubmed: https://www.ncbi.nlm.nih.gov/pubmed/30622526

82. Zhu L, Liu L, Zhang Y, Pu L, Liu J, et al. High level of neutrophil extracellular traps correlates with poor prognosis of severe influenza A infection. J Infect Dis. 2018; 217: 428-437.

Pubmed: https://www.ncbi.nlm.nih.gov/pubmed/29325098

83. Narasaraju T, Yang E, Samy RP, Ng HH, Poh WP, et al. Excessive neutrophils and neutrophil extracellular traps contribute to acute lung injury of influenza pneumonitis. Am J Pathol. 2011; 179: 199-210. Pubmed: https://www.ncbi.nlm.nih.gov/pubmed/21703402

84. Hosseinzadeh A, Thompson PR, Segal BH, Urban CF. Nicotine induces neutrophil extracellular traps. J Leukoc Biol. 2016; 100: 1105-1112. Pubmed: https://www.ncbi.nlm.nih.gov/pubmed/27312847
85. Taghizadeh $F$, Akbari $H$. The powerful immune system against powerful COVID-19: A hypothesis. Med Hypothesis. 2020.

86. Yuki K, Fujiogi M, Koutsogiannaki S. COVID-19 pathophysiology: A review. Clin Immunol. 2020: 108427.

Pubmed: https://www.ncbi.nlm.nih.gov/pubmed/32325252

87. Zuo Y, Yalavarthi S, Shi H, Gockman K, Zuo M, et al. Neutrophil extracellular traps in COVID-19. JCI Insight. 2020. 138999.

Pubmed: https://www.ncbi.nlm.nih.gov/pubmed/32329756

88. Bendib I, de Chaisemartin L, Mekontso Dessap A, Chollet-Martin S, de Prost N. Understanding the role of neutrophil extracellular traps in patients with severe pneumonia and ARDS. Chest. 2019; 156: 12781280.

Pubmed: https://www.ncbi.nlm.nih.gov/pubmed/31812204

89. Geerdink RJ, Pillay J, Meyaard L, Bont L. Neutrophils in respiratory syncytial virus infection: A target for asthma prevention. J Allergy Clin Immunol. 2015; 136: 838-847.

Pubmed: https://www.ncbi.nlm.nih.gov/pubmed/26277597

90. Domingo-Gonzalez R, Martínez-Colón GJ, Smith AJ, Smith CK, Ballinger MN, et al. Inhibition of neutrophil extracellular trap formation after stem cell transplant by prostaglandin E2. Am J Respir Crit Care Med. 2016; 193: 186-197.

Pubmed: https://www.ncbi.nlm.nih.gov/pubmed/26417909

91. te Velthuis AJ, van den Worm SH, Sims AC, Baric RS, Snijder EJ, et al. $\mathrm{Zn}^{2+}$ inhibits coronavirus and arterivirus RNA polymerase activity in vitro and zinc ionophores block the replication of these viruses in cell culture. PLoS Pathog. 2010; 6: e1001176.

Pubmed: https://www.ncbi.nlm.nih.gov/pubmed/21079686 